\title{
Trajectoires d'innovation en agriculture de conservation au lac Alatra à Madagascar
}

\section{Trajectories of innovation in agriculture of conservation at Lake Alatra in Madagascar}

\author{
Eric Penot ${ }^{1}$, Valentin Fevre \& Patricia Flodrops ${ }^{2}$ \\ 1 CIRAD UMR Innovation, Montpellier, eric.penot@cirad.fr \\ 2 AgroParisTech/UMR Innovation, Paris, valentin.fevre@agroparistech.fr, patricia.flodrops@agroparistech.fr
}

RÉSUMÉ. La région du Lac Alaotra est un des greniers à riz de Madagascar avec de nombreuses contraintes pédoclimatiques importantes. Dans une perspective de développement durable, des organismes de recherche ont mis au point des itinéraires techniques issus de l'Agriculture de Conservation (AC).La dissémination a été assurée de 2003 à 2013 par le projet de développement BV-Lac. Comme définie par la FAO en 2008, l'AC répond à trois principes : i) perturbation minimale du sol, ii) protection du sol par une couverture végétale, iii) rotation de culture et association de plantes qui impliquent un double changement de paradigme sur les techniques de cultures et les stratégies. Une enquête réalisée en 2013/2014 montre que depuis le démarrage du projet, les paysans ont su adapter ces techniques à la structure de leur exploitation pour répondre à leurs propres contraintes dans des processus d'appropriation aboutissant un continuum de pratiques entre conventionnel et AC mettant en exergue un long temps d'apprentissage et une recombinaison des savoirs.

ABSTRACT. Alaotra Lake area is one of Madagascar's most important rice growing regions, with important soil and climatic constraints. Through sustainable agricultural development, the BV-lac project has disseminated techniques of conservation agriculture (CA), between 2003 to 2013. As defined by the FAO in 2008, CA has three principles: i) minimum soil disturbance, ii) soil protection by vegetation cover, iii) crop rotation and plant association, requiring a double paradigm shift on cropping pattern and on strategies. A survey conducted in 2013/2014 showed that during the project, farmers adapted CA techniques to their own constraints in a long learning process leading to a continuum of practices between conventional agriculture and CA through a recombination of knowledge.

MOTS-CLÉS. Agriculture de conservation, processus d'innovation, stratégies paysannes, lac Alaotra, Madagascar.

KEY WORDS. Conservation Agriculture, innovation processes, farmers' strategies, Alaotra lake, Madagascar.

\section{Introduction}

La région du Lac Alaotra (carte $\left.\mathrm{n}^{\circ} 1\right)$ est considérée comme le grenier à riz de Madagascar avec de nombreuses contraintes pédoclimatiques (érosion importante y compris d'ordre géologique, pression sur les ressources naturelles par la pression démographique...) (DURAND et al., 2008). Pour stabiliser les productions et augmenter la fertilité des sols, des organismes (CIRAD, FOFIFA, TAFA) ont mis en place des activités de recherche pour mettre au point des itinéraires techniques issus de l'Agriculture de Conservation $(\mathrm{AC})^{1}$. La dissémination à large échelle a été faite auprès des agriculteurs de 2003 à 2013 par le projet de développement BV-Lac (sur financement AFD/Agence Française de Développement).

\footnotetext{
${ }^{1}$ Ou Systèmes de culture sous Couvert Végétal (SCV) dont la définition est équivalente.
} 
Comme définie par la $\mathrm{FAO}$ en 2008, l'AC répond à trois principes : i) perturbation minimale du sol, ii) protection du sol par une couverture végétale, iii) rotation de culture et association de plantes.

L'objectif initial de ces systèmes de culture en AC est de stabiliser les productions, de préserver les ressources naturelles vers une agriculture durable et d'augmenter la fertilité des sols en relançant leur vie biologique (SCOPEL et AL. 2004, SCOPEL et al., 2012). Si ces systèmes sont prometteurs, il existe cependant des contraintes importantes à leur adoption qui ont été observées dans différents pays d'Afrique sub-saharienne (GILLER et al., 2009; SERPANTIE, 2009, CORBEELS et al, 2014). L'approche initiale préconisée par un groupe d'agronomes était clairement de type diffusionniste (ROGER 1962). L'équipe du projet BV-lac a rapidement compris que cette approche était dépassée, inopérante et a développé des outils de suivi et de compréhension des stratégies paysannes pour intégrer en temps réel : i) les processus d'innovation en cours (réseau de fermes de références, approche exploitation, analyse prospective etc ...), ii) les formes d'appropriation (techniques et aussi sociales) et iii) les modalités d'apprentissage long afin de tenter d'adapter d'une part les recommandations techniques du projet aux réalités paysannes locales et, d'autre part, d'utiliser des méthodes de dissémination plus adaptées intégrant par exemple le conseil à l'exploitation (FAURE et al., 2012). Le temps de l'innovation n'est pas le plus souvent pas celui des projets (PENOT et al. 2014).

Cette nouvelle approche nécessitait d'intégrer les stratégies paysannes locales, de mesurer l'impact du changement technique au niveau de l'exploitation agricole et de développer des formes de conseil à l'exploitant plus large que la simple diffusion d'un modèle technique. L'augmentation régulière du nombre apparent «d'adoptants » et des surfaces en AC suivies par le projet ainsi que la forte demande d'appuis et de conseils pour l'agriculture de conservation a montré un succès relatif (DOMAS et al. 2009) pendant la durée du projet. Depuis le démarrage du projet, les enquêtes de suivi et plus particulièrement celles faites après 2006 intégrant l'impact de l'adoption complète ou partielle de ces techniques sur le fonctionnement des exploitations agricoles ont clairement montrées une capacité très forte d'innovation des producteurs (GARIN et PENOT 2011), une adaptation des techniques aux contraintes locales très diverses et une logique d'appropriation impliquant un long apprentissage de 7 à 10 ans pour intégrer complétement, ou pour la plupart des paysans, partiellement ces techniques (PENOT et al., 2015). Le projet BV-Lac est arrivé à terme en juin 2013.

La question de la durabilité de ces actions de développement et de l'adoption/appropriation sous des formes techniques diverses à long terme des techniques de l'Agriculture de Conservation au lac Alaotra peut donc être en partie évaluée. Ainsi, l'analyse des perceptions paysannes sur un échantillon particulier (celui des plus anciens adoptants ou considérés comme tels à la fin du projet) permet de comprendre si ces techniques répondent bien à des contraintes locales majeures, si leur adoption a pu améliorer la résilience des systèmes productifs (PENOT et al., 2014) et sous quelles modalités d'adaptation/appropriation les systèmes techniques ont évolués. On est passé d'une approche sectorielle (et au début assez dirigiste) à un système d'innovation régional plus complexe et intégré. Finalement la nécessité du changement de paradigme de l'agriculture conventionnelle à l'AC a fait changer autant les paysans utilisateurs qui hybrident par nature les savoirs pour en développer de nouvelles pratiques que les agents du projet qui apportent un savoir et des techniques et les font aussi évoluer dans le temps en fonction du suivi réalisé. Cette boucle (feedback) sur les processus d'innovation a permis la reconnaissance des formes d'apprentissage long et le développement d'outils plus adaptés comme le conseil à l'exploitation. 


\section{Problématique}

L'agriculture de conservation est basée sur 3 principes de base comme le définit la FAO en 2008 : i) un minimum de perturbation du sol et donc par définition le non labour, ii) l'utilisation d'une plante de service pour la décompaction des sols et la création d'une biomasse importante utilisée comme mulch et iii) une rotation des cultures incluant les plantes de service.

Il existe en 2012 un cour d'adoptants (410 ha pour 600 paysans en 2010, estimé à 700/800 paysans, PENOT et al.,2011), c'est-à-dire un nombre de paysans qui ont théoriquement définitivement adopté les techniques en $\mathrm{AC}$ avec un changement de paradigme qui suppose une conversion à la fois technique (par l'abandon du labour) et comportementale (passage d'un raisonnement tactique, à court terme, à un raisonnement stratégique en pensant le système de culture à long terme). Il paraît peu envisageable que ce « cœur d'adoptants » puisse permettre aux techniques en AC de diffuser spontanément à d'autres producteurs non encadrés comme le montre l'enquête réalisée par A TEISSONIER en 2013. Des systèmes de culture intégrant des techniques en AC sans en adopter la totalité, semblent être adoptés à plus large échelle et seraient la conséquence d'une diffusion spontanée d'une partie seulement du paquet technique complet des techniques en AC.

Ces systèmes ont été appelés Systèmes de Culture Innovants ou SCI (FABRE 2011) car ils illustrent la recombinaison des savoirs et le caractère hybride des systèmes techniques finalement développés par les paysans après apprentissage. Le processus d'innovation intègre le temps long et un apprentissage en plusieurs étapes comme nous allons le voir.

La problématique globale est donc celle de l'évolution des systèmes de culture entre leur introduction/dissémination par un projet de développement "classique» et ce que les paysans finalement en font. On cherche à comprendre les modalités d'adoption/appropriation des techniques de l'agriculture de conservation par les producteurs familiaux de la région du lac Alaotra intégrant des conditions post-projet, sans aide technique si support de quelque nature. Si le chercheur et le développeur voient en l'AC un double paradigme, le producteur voit une opportunité d'une part de stabiliser sa production agricole en mode pluvial sur des sols fragiles au prix d'un changement de pratiques, voire même de l'augmenter à moindre risque au prix d'une certaine intensification en intrants. La perception paysanne de l'innovation est donc essentielle pour comprendre le processus d'évolution. 


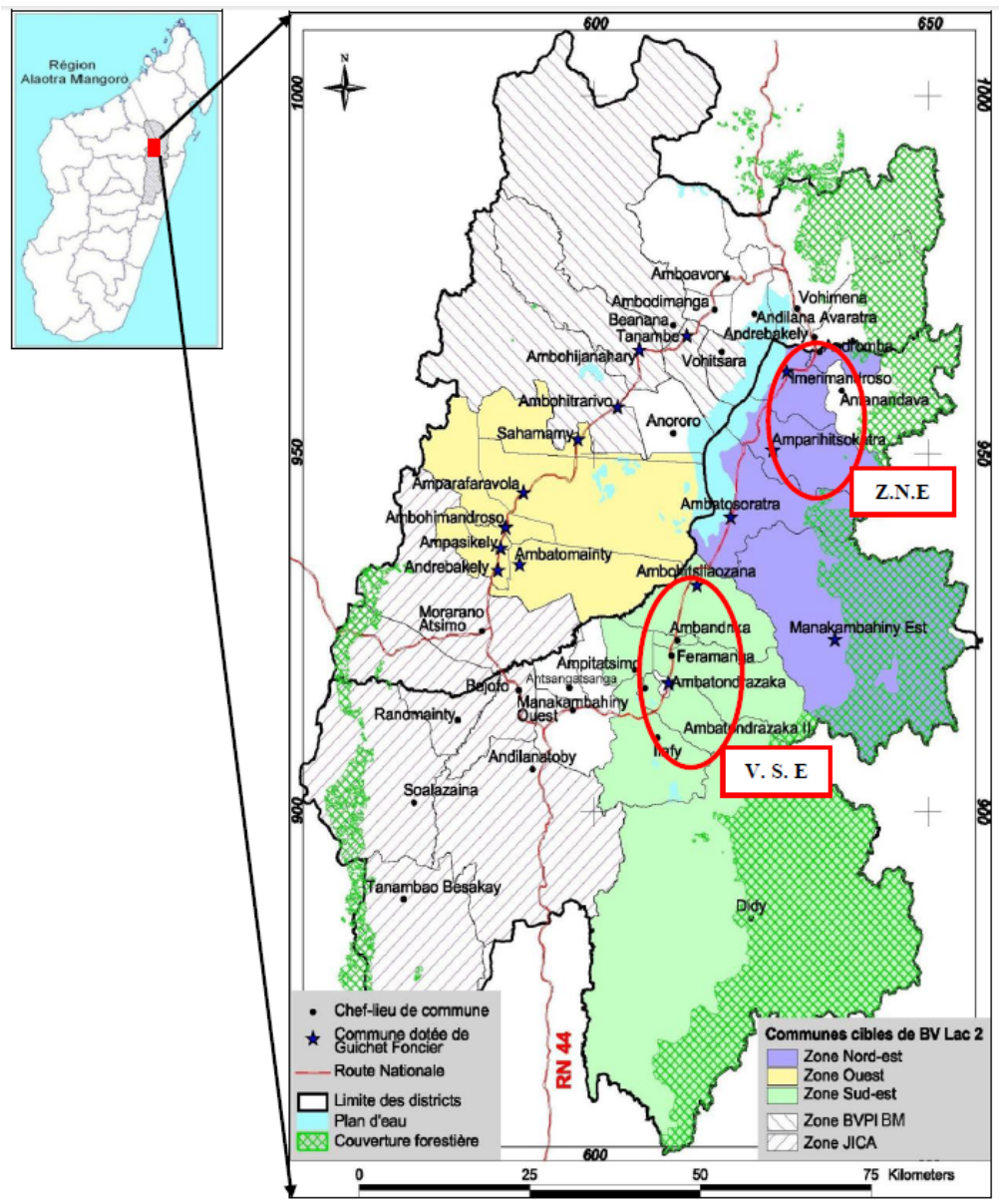

Carte n 1. Le lac Alaotra à Madagascar et zones d'études (ZNE/Zone Nord-Est et VSE/ Vallées du Sud Est)

L'objectif de cette étude est de voir: i) l'évolution des systèmes de culture en AC adoptés et transformés par les paysans du projet BV-Lac et les innovations apportées sur 10 ans, ii) d'identifier le temps nécessaire à leur intégration complète dans les exploitations, les modalités de leur évolution en fonction des formes d'apprentissage et d'appropriation des techniques et, iii) de déterminer quels sont les éléments d'adoption des techniques en AC, les conditions sociales de l'adoption et l'évolution des trajectoires. Le comportement de ces «anciens adoptants » en condition post-projet est également analysé.

\section{Méthodologie}

Cette analyse s'appuie sur des enquêtes auprès d'agriculteurs (104 enquêtés en direct par des entretiens individuels et 28 via des focus groupe) situés dans la zone nord-est (ZNE) et dans la vallée du sud-est (VSE) du lac qui s'opposent d'un point de vue géographique, social et d'intégration au marché ${ }^{2}$ L'échantillonnage est centré sur les parcelles de 6 à 10 ans avec des producteurs les plus

\footnotetext{
${ }^{2}$ Cette étude poursuit en partie le travail mené en 2009 par Raharisoa en reprenant les paysans déjà enquêtés à cette date pour analyser l'évolution de leurs systèmes depuis 10 ans et les éventuels abandons.
} 
anciens du projet qui étaient considérés comme des adoptants « définitifs » en 2013 à la fin du projet sur la base d'une première enquête finalisée en 2009 (PENOT et al. 2014).

L'analyse des résultats par parcelle a permis d'identifier l'évolution des itinéraires techniques paysans par rapport aux recommandations initiales et les raisons des abandons post-projet. Une typologie de situation et une autre de comportement des producteurs a permis de comprendre les trajectoires d'évolution des principaux systèmes de culture en $\mathrm{AC}$ et les logiques d'appropriation. La démarche globale est synthétisée dans la figure $\mathrm{n}^{\circ} 1$.

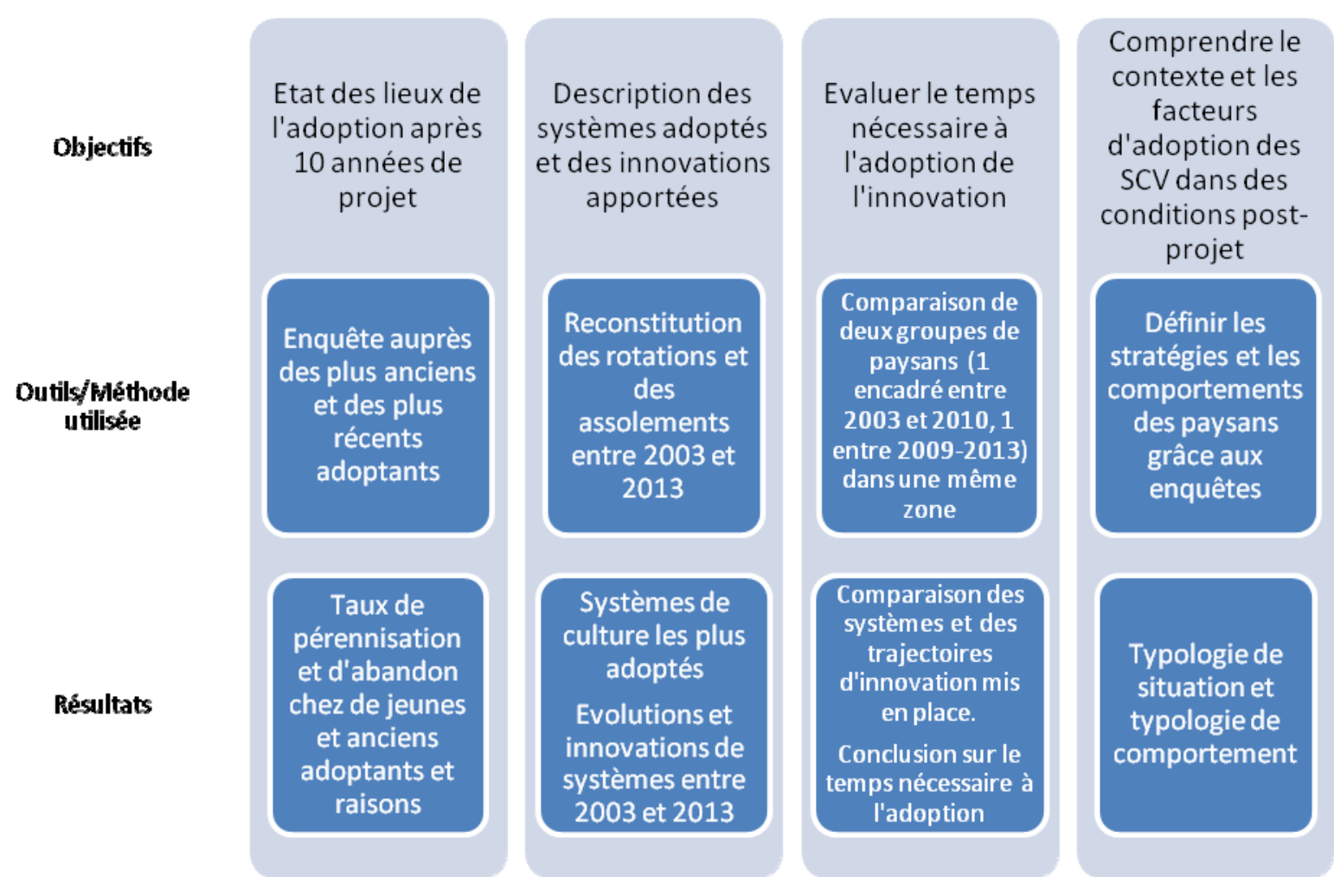

Figure 1. Synthèse de la démarche méthodologique

\section{Etat des lieux sur l'adoption de l'agriculture de conservation en 2013}

Le projet BV-lac a duré 10 années : ceci est beaucoup pour un projet mais peu pour l'apprentissage nécessaire à un véritable changement de paradigme (de l'agriculture conventionnelle à l'AC). En 2014, un fort taux d'abandon des techniques de l'agriculture de conservation $(40 \%)$ est observé chez les « anciens adoptants» (plus de 7 ans) ${ }^{3}$, ce qui semble confirme l'hypothèse selon laquelle minimum 5 ans d'ancienneté dans les pratiques sont nécessaires pour adopter définitivement les techniques en AC et probablement entre 2 et 3 années supplémentaires pour adapter localement les techniques AC (incluant une adoption partielle des différentes thèmes techniques). Les paysans de la zone nord ont abandonné

\footnotetext{
${ }^{3}$ ) Comme chez les « jeunes » adoptants (moins de 5 ans) qui ne sont pas compris dans notre analyse 
plus massivement (55\%) que ceux du sud (20\%). Cela est probablement dû à la difficulté à mettre en place des techniques AC sur les tanety en condition pluviales (DOMAS et al., 2009), (bien plus nombreux dans le nord que dans le sud) et à l'individualisme plus important des paysans du nord (faible cohésion sociale qui entraîne des divagations des animaux et moins d'entraide). Les blocages sont souvent d'ordre économique (pour 60\% des anciens adoptants) et social (57\%). Les difficultés purement techniques ne sont donc pas les premières en cause (mise en place de mulch, de cultures associées, gestion des rotations, contrôle de l'enherbement etc ...

La première raison d'abandon citée par 33\% des anciens encadrés est l'augmentation des dépenses qui concerne l'achat des produits phytosanitaires ainsi que les besoins en main d'œuvre journalière pour la préparation du sol, la plantation, le transport de la couverture (pour les systèmes à couverture morte initialement promus puis rapidement abandonnés). Les premiers systèmes de culture en AC étaient promus avec un certain niveau d'intensification en engrais et traitements phytosanitaires pour permettre un réel saut significatif de rendement. La crise de 2008 et le doublement du prix des intrants a stoppé net ce début de processus d'intensification et à partir de cette date, tous les itinéraires techniques en $\mathrm{AC}$ sont devenus à très faible niveau d'intrants et, donc, avec une très faible augmentation de la production. Il n'y a pas ou peu d'économie de temps de travaux effectivement ressentie comme telle par les paysans (en particulier pour les systèmes à base de Styloxanthes spp), ni de capital comme cela l'avait été originellement présenté aux paysans. Seuls les systèmes à base de dolique et de vesce (dans les baiboho ou sols colluviaux) ont générés des économies de main d'œuvre, En effet les techniques en $\mathrm{AC}$ en elle mêmes n'augmentent pas significativement les rendements comme le font les engrais organiques ou minéraux mais par contre par l'effet mulch contribuent à une plus grande efficience de ces intrants quand ils sont utilisés. L'arrêt du projet (entraînant l'absence du technicien, l'arrêt des aides éventuelles et augmentant la divagation des zébus) est déterminant chez les anciens adoptants pour justifier leur abandon ce qui est pour le moins paradoxal puisque ce sont eux qui ont profité le plus longtemps de l'appui des techniciens.

Le manque de cohésion sociale des groupes de paysans et leur incapacité à suivre des règles communautaires simples sont globalement responsables en partie de la non-durabilité des techniques SCV au lac parmi lesquelles deux exemples sont significatifs : i) la divagation non contrôlée des animaux en situation post-projet (qui implique la destruction des mulchs en hivernage) et ii) le difficile accès au crédit à caution solidaire après 10 années d'expérimentation (qui limite l'intensification des systèmes de cultures). Ces deux exemples flagrants montrent la nécessaire intégration de l'évolution du tissu social dans la compréhension des stratégies paysannes. Finalement, seulement $53 \%$ des « anciens » encadrés forment le « cœur d'adoption » en 2014 (sur notre échantillon de 80 exploitants).

Les principales raisons d'abandon sont par ordre d'importance : i) l'augmentation des dépenses pour les intrants (cité par 33\% des paysans ayant abandonné), ii) l'absence du technicien (23\%) qui est directement liée à l'arrêt du projet, iii) la divagation des zébus $(23 \%)$ est redevenue un problème majeur à l'arrêt du projet, iv) l'augmentation des ravageurs (23\%), v) l'absence d'aides et le difficile recours au crédit (23\%) (L'arrêt des crédits est dû à l'absence des remboursements des prêts à caution solidaire par certains membres des groupements de producteurs et vi) la sécheresse (20\%) et les risques de culture du fait du caractère erratique des pluies apparaissent aussi comme une raison importante d'abandon, en particulier si le mulch n'est pas suffisamment développé. 
Face à ce fort taux d'abandon post projet pour un échantillon de paysan très particulier considéré comme le cœur d'adoption du projet, on peut se demander si les techniques AC sont réellement adaptées aux contraintes locales, et aussi quelle est la compréhension réelle et la perception des paysans sur ces techniques. L'enquête a permis d'identifier une typologie de situation. Trois grands types de situation sont distingués dans le tableau 2, selon la dynamique d'évolution de l'adoption de l'AC au sein de l'exploitation. Des sous-types distinguent les exploitations par rapport à l'importance accordée à l'AC.

\begin{tabular}{|c|c|c|c|c|}
\hline $\begin{array}{l}\text { Evolution des } \\
\text { surfaces en AC } \\
\text { depuis } 2009\end{array}$ & $\begin{array}{c}\text { Surface en AC/surface } \\
\text { potentielle }\end{array}$ & $\begin{array}{c}\text { Surface en AC/ surface } \\
\text { totale }\end{array}$ & Effectif & $\begin{array}{l}\text { Type de } \\
\text { situation }\end{array}$ \\
\hline \multirow{5}{*}{ Augmentation } & \multirow{2}{*}{$>100 \%$} & $>50 \%$ & $3 \%$ & I1 \\
\hline & & $<50 \%$ & $3 \%$ & I2 \\
\hline & $100 \%$ & entre $35 \%$ et $80 \%$ & $8 \%$ & I3 \\
\hline & \multirow{2}{*}{$<100 \%$} & entre $25 \%$ et $60 \%$ & $7 \%$ & I4 \\
\hline & & $<25 \%$ & $9 \%$ & I5 \\
\hline \multirow{3}{*}{ Pas d'évolution } & $>75 \%$ & $<20 \%$ & $4 \%$ & III \\
\hline & entre $25 \%$ et $75 \%$ & $<25 \%$ & $3 \%$ & II2 \\
\hline & $<25 \%$ & $<20 \%$ & $12 \%$ & II3 \\
\hline \multirow{4}{*}{ Diminution } & \multirow{2}{*}{$>60 \%$} & $100 \%$ & $1 \%$ & III1 \\
\hline & & $<35 \%$ & $7 \%$ & III2 \\
\hline & entre $5 \%$ et $30 \%$ & $<20 \%$ & $7 \%$ & III3 \\
\hline & $0 \%$ & $0 \%$ & $38 \%$ & III4 \\
\hline
\end{tabular}

Tableau 2. Typologie de situation des exploitations

\section{Evolutions des pratiques paysannes et des innovations en agriculture de conservation.}

Le terme " grand système de culture » désigne les systèmes en AC à base d'une plante de service. Cinq grands systèmes ont été diffusés : à base de dolique, de Brachiaria spp, de Stylosanthes spp, de vesce et de couverture morte. Différentes modifications de ces systèmes ont été observées et les principaux systèmes sont présentés dans cette partie, ainsi que leur évolution.

Sur les parcelles de tanety, initialement étudiées de 2003 à 2009 par RAHARISOA, deux grands systèmes sont majoritaires de 2009 à 2013 : le « système à base de résidus de culture » et le système " maïs+légumineuses volubiles » dont les pourcentages varient peu durant ces années (respectivement $40 \%$ et $32 \%$ des surfaces non abandonnées en 2013). Le système à base de résidus (avec souvent riz et arachide sur paillage) permet de valoriser les restes de la culture précédente comme mulch. Le système 
« maïs+légumineuse volubile type dolique », préconisé par les techniciens, est un système à base de couverture vive qui permet normalement le renouvellement de la biomasse. Cependant, même si la dolique est préconisée avec le maïs car elle offre facilement un bon mulch, elle n'est pas valorisable économiquement contrairement au niébé (Vigna unguiculata). De ce fait, le niébé est la plante la plus souvent associée avec le maïs entre 2004 et 2013 (avec aussi le «rice bean » Vigna umbellata) mais il ne procure pas un mulch important. Dans tous les cas, une diversification des systèmes est remarquable sur les topo-séquences chez ces anciens paysans. Il est à noter qu'à partir de 2010, les systèmes encore présents sont relativement stables d'une année sur l'autre.

Pendant les 7 premières années de pratique, les paysans testent les systèmes préconisés par le projet BV-Lac. Les systèmes préconisés évoluent également au sein du projet par l'intégration des diverses modification paysannes observées par les techniciens et analysées annuellement, ce qui a abouti à une évolution dans les préconisations du projet. Le projet a donc bien réagi en fonction des pratiques paysannes pour adapter les itinéraires techniques afin de maximiser leur adoption potentielle. Cette démarche d'intégration s'inscrit dans la démarche exploitation adoptée par le projet depuis 2008. Après 7 ans, les paysans pratiquant l'AC ont dépassé la phase d'expérimentation et semblent conserver quelques grands systèmes adaptés à leurs besoins.

A partir de 2010, un fort taux de SCI est observé (de 4\% à 15\% des surfaces selon la topo-séquence et l'année d'entrée en SCV). Les SCI constituent une innovation paysanne, à travers une évolution technique de simplification des systèmes de culture SCV. L'absence du technicien et de ses conseils sur la parcelle est sans doute déterminante pour l'apparition des SCI car le paysan a moins tendance à suivre « à la lettre » les préconisations donnée. Le projet a donc joué le rôle de forme sociale structurante, du moins pendant la durée effective de présence des techniciens sur le terrain. L'apparente cohésion sociale pendant le projet s'est effondrée à la fin du projet. La figure 2 montre l'évolution des grands systèmes de culture entre 2002 et 2013 sur les anciennes parcelles de tanety. 


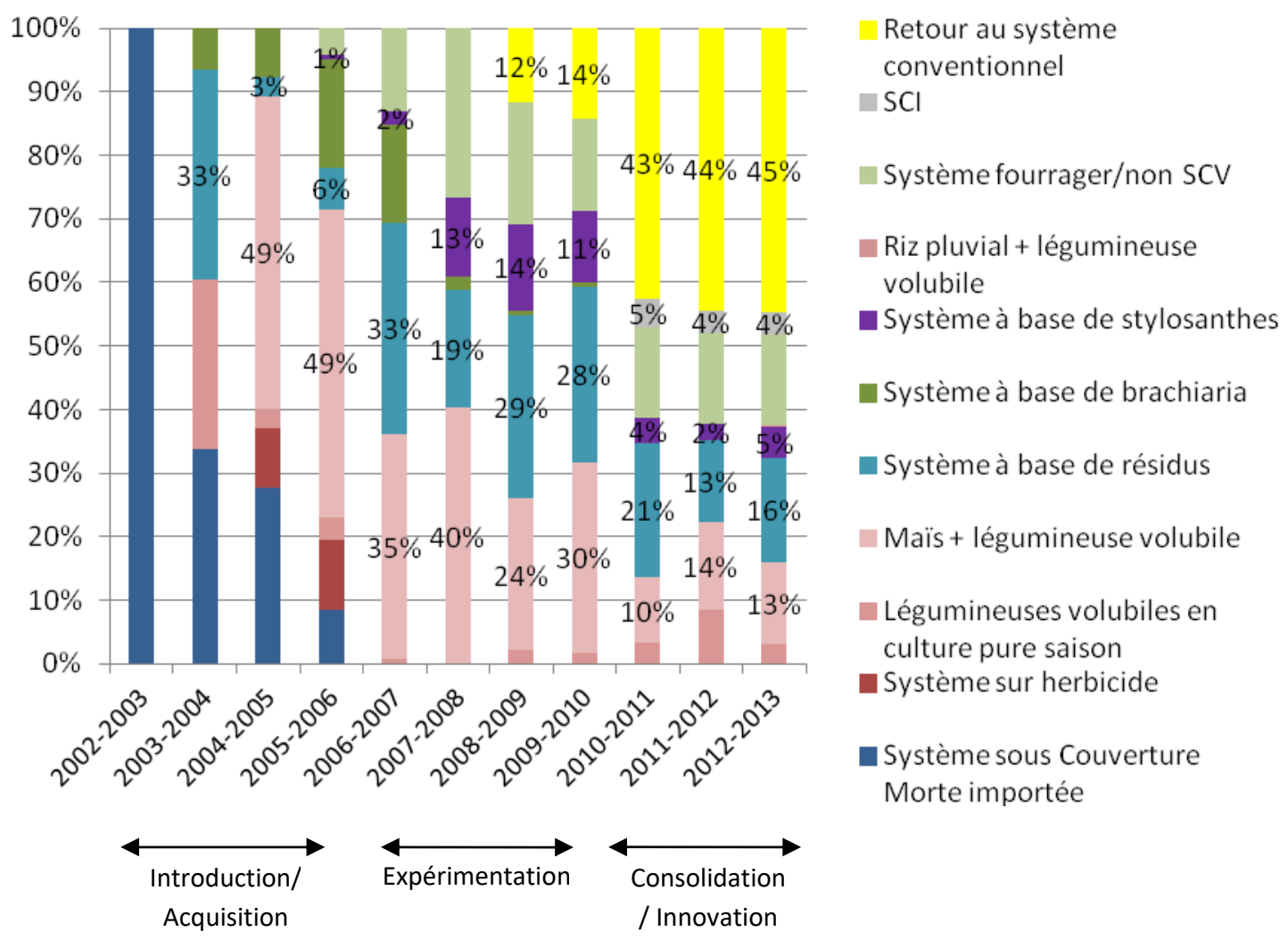

Figure 2. Evolution d'adoption des grands systèmes sur anciennes parcelles de tanety

\section{Des systèmes de culture très diversifiés chez les adoptants les plus anciens.}

Les systèmes majoritairement mis en place sur tanety par les plus anciens adoptants sont la rotation " maïs+légumineuse//riz pluvial//arachide » dans le nord (43\% des surfaces) et le " système continu de maïs » dans le sud $(46 \%)^{4}$. Dans ce système, $70 \%$ des surfaces sont en système continu « riz / haricot ou petit-pois + vesce ». On constate une explosion de divers itinéraires techniques avec par exemple sur tanety 5 « nouveaux » systèmes en 2014 non identifiés en 2009. L'innovation paysanne s'est donc poursuivie ces dernières années et a eu pour principal objectif d'intensifier la production globale pour augmenter les revenus : priorité à la production de riz et de maïs le plus souvent en association avec haricot, introduction de l'arachide dans la rotation (avec pour conséquence un très faible mulch), substitution de la dolique par des plantes valorisables économiquement mais produisant beaucoup moins de mulch... Mais l'intensification de la production (par le nombre de cultures rémunératrices) se fait souvent au dépend de la couverture obligeant un labour plus fréquent pour décompacter le sol et pour lutter contre les adventices puisque la plante de service ne remplit plus ces rôles correctement. On observe alors un glissement des SCV stricto sensu vers des systèmes SCI ou proches des SCI. Ainsi, $70 \%$ des systèmes de culture sur tanety ne sont plus des systèmes en AC à proprement parlé mais tendent vers des SCI.

\footnotetext{
${ }^{4}$ Sur baiboho, c’est le système continu « riz/système vesce » qui domine ( $41 \%$ des surfaces totales) 
La figure 3 (fin de texte) présente l'évolution des systèmes de culture et des divers itinéraires techniques depuis leur diffusion jusqu'en 2012-2013. On remarque une grande diversification des systèmes depuis montrant que les paysans ont largement innovés sur les systèmes initialement diffusés et les ont adaptés à leurs objectifs de production. La figure 4 montre l'évolution des surfaces ayant débutées par une légumineuse volubile en culture pure ou associée au mais sur tanety. Ces éléments montrent la très grande diversité des systèmes. Finalement on observe un quasi continuum entre systèmes en AC stricto sensu, systèmes SCI et système conventionnel (MAC DOWALL, 2011).

Même si les techniques en $\mathrm{AC}$ ne permettent pas une réelle augmentation des rendements (sans intensification concomitante par les engrais), elles permettent néanmoins aux paysans d'assurer une production et de produire lorsque les autres systèmes en conventionnel ne le peuvent pas (sécheresse en début de cycle par exemple, protection du mulch,...). Si les paysans ont globalement peur d'investir massivement dans un système si différent et ne procurant des avantages significatifs que sur le moyen terme, ils sont cependant extrêmement sensibles à tout ce qui peut améliorer la résilience du système de culture. Les effets positifs des mulchs sont maintenant bien connus et recherchés. Mais la formation d'un bon mulch reste difficile : les "plantes de service » choisies ne sont pas toujours adaptées au type de sol et contribuent à une certaine compaction des sols après 4 ou 5 années. Une observation fine des parcelles à dire d'expert sur les 10 années mais non mesurées statistiquement permet d'observer que 50 $\%$ des mulchs sont insuffisants dans les systèmes en AC pour avoir les effets attendus. L'innovation créée par les paysans à travers la richesse des SCI est très intéressante. Il serait souhaitable que l'on puisse suivre ces évolutions à moyen terme pour améliorer la capacité à proposer des nouveaux systèmes plus adaptés (intégrant l'arachide par exemple...). Le projet a cependant faite une analyse prospective (COTTET et PENOT 2010) sur l'ensemble des 130 itinéraires techniques observés en AC avec divers modes de valorisation par l'élevage par exemple, sur la base des modes d'appropriation observés et des transformations des systèmes effectuées par les producteurs pour identifier les systèmes les plus prometteurs à travers une véritable démarche de Recherche Action en Partenariat (RAP) (DULCIRE et al., 2008).

\section{Apprentissage, co-conception des innovations et système d'innovation}

\subsection{Apprentissage et recombinaison des savoirs}

La figure 5 illustre le processus d'apprentissage, d'adaptation puis d'appropriation des techniques AC observés avec les paysans suivis par le projet. 


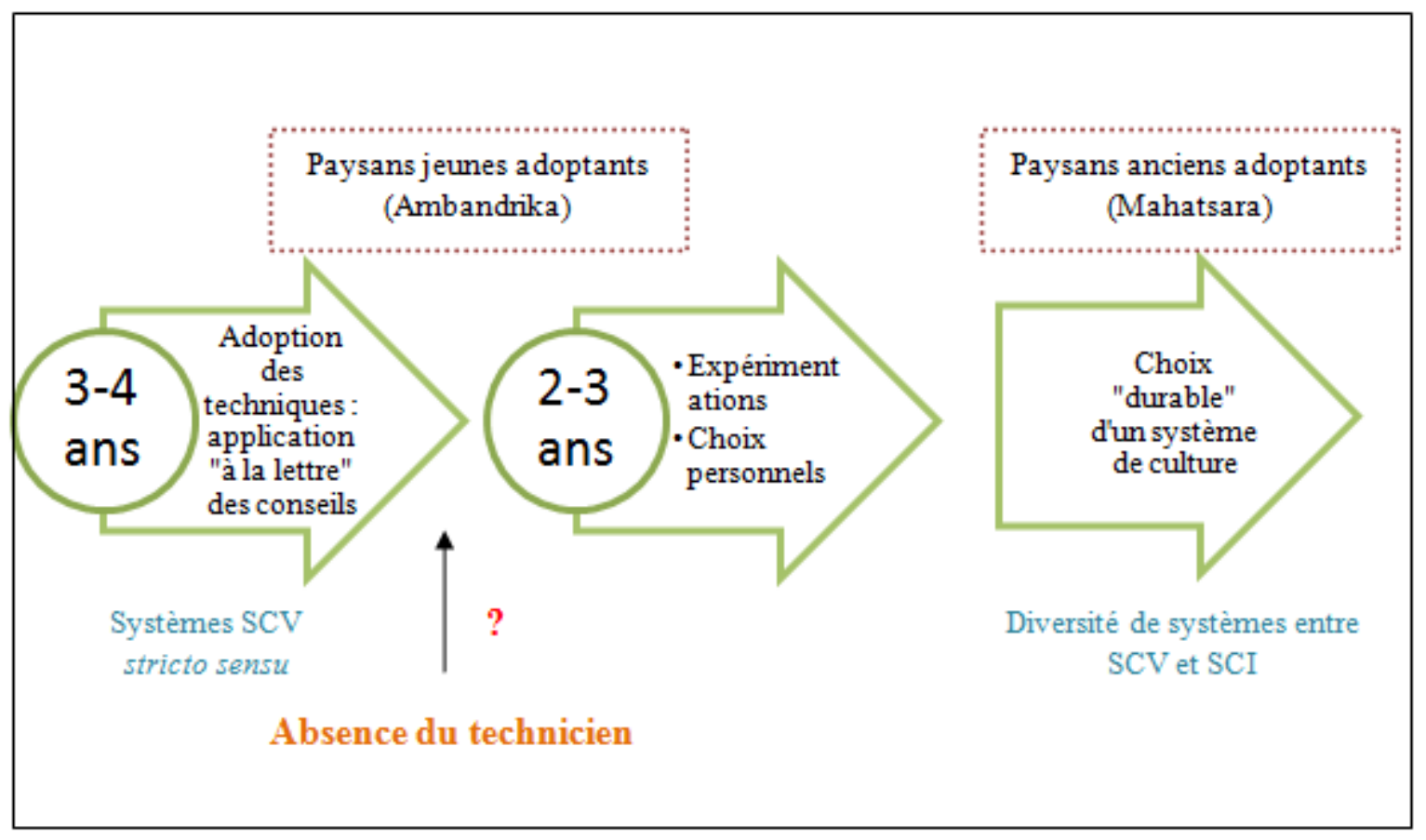

Figure 5. Schéma d'adoption de l'innovation

Les formes d'apprentissage sont essentiellement individuelles. Le manque de confiance généralisé dans les institutions hors de la famille en général et la dégradation des règles sociales dans la vie des villages (vols) a très fortement limité toutes les actions collectives (URFER 2014). L'analyse des actions du projet sur le développement des Organisations Paysannes (OP) a montré que seuls 8 OP sur les 85 suivies et aidées par le projet étaient viables et actives en 2012 ce qui montre clairement que la structuration des producteurs est extrêmement difficile dans le contexte malgache comme l'avait montré Fouroux dans son article sur «l'illusion participative » (BLANC-PAMARD et FOUROUX 2004).

\subsection{Un exemple empirique d'évolution vers la co-construction des systèmes}

Finalement le type de système d'innovation (SI) auquel on se réfère dans cette situation relève du quatrième groupe identifié par TOUZARD et al., en 2015: le groupe des acteurs impliqués dans l'action et la mise en place de processus d'innovation techniques (AC) et organisationnels (OP et crédit) mais dans une évolution concomitante d'un part des producteurs à la recherche de solutions techniques et organisationnelles compatibles avec leur situation et leur structure sociale et d'autre part un projet de développement qui a intégré un passage progressif d'une approche initiale centrée sur le «top-down» à une conception des systèmes intégrant pleinement les adaptations de ces mêmes producteurs. Il ne s'agit pas d'une co-conception voulue dès le départ mais d'une co-évolution vers un partenariat ou les acteurs de la production sont mieux reconnus, une situation finalement assez originale dans le concept malgache. Le mécanisme de reconnaissance de l'hybridation des techniques et des pratiques est ainsi devenu institutionnel, du moins dans le cadre du projet et pendant sa durée.

Le rapport sur la recherche de solutions aux contraintes fortes et multiples de nature pédoclimatiques, environnementales, économiques et sociales entre les acteurs s'en est ainsi trouvé modifiée et le passage à la mise en place d'un conseil de gestion, même embryonnaire y a largement 
contribué. Si on n'est pas encore dans la production de « biens publics » basés sur les externalités des systèmes en $\mathrm{AC}$, la reconnaissance de la capacité d'innovation locale a clairement favorisé certaines pratiques ayant un important impact environnemental pourtant issues d'un changement de paradigme profond pour les populations locales. La complexité des enjeux en cours de diverses natures: agriculture durable, préservation des ressources, structuration des producteurs, réhabilitation du collectif dans un monde socialement et politiquement incertain a renforcé la nécessité d'une approche multidisciplinaire intégrant le moyen et le long terme (COUDEL et al., 2012).

\subsection{Vers une co-gestion de l'innovation}

L'analyse des pratiques et leurs trajectoires d'évolution sur 10 ans montrent bien que finalement les paysans locaux ont profité partiellement d'un dispositif de production devenu cognitif avec les acteurs engagés (DULCIRE 1996) au fil du temps alors que l'approche initiale était clairement dirigiste, orientée et assez paternaliste. Comme le rappelle Dulcire et al «Ce processus commun aux chercheurs et acteurs de terrain garantit la durabilité des actions ». Il a en tous cas généré un certain niveau de durabilité par rapport à d'autres expériences de «transfert » totalement ratée, comme celle développée par l'ONG TAFA dans les années 1996/2002 à Ivory dans le Moyen Ouest du Vakinanakaratra ${ }^{5}$. La transition agro-écologique fait prendre un certain nombre de risques aux producteurs dans un contexte d'incertitudes déjà multiples. Les éléments techniques qui concourent de façon significative et surtout visible à court terme à une meilleure résilience des systèmes de cultures sont ceux qui sont le plus facilement durablement adoptés.

Finalement, les différentes outils mis en place par le projet (fermes de références, analyse prospective, sessions de partage de l'innovation, conseil à l'exploitation, parcelles de démonstrations, visites inter-villages...) ont abouti à la création d'un partenariat ou le paysan n'est plus considéré comme une simple cible pour la diffusion et, donc, un futur « adoptant» (terme réducteur s'il en est !), mais comme un partenaire puisque on prend en compte son avis, sa trajectoire et les pratiques différenciées qui découlent de la mise en œuvre des techniques. Cette co-construction au final des systèmes, du moins dans les 4 dernières années du projet, a été faite au prix d'un très lourd investissement dans la formation des techniciens et ingénieurs du projet.

Si l'agriculture de conservation est manifestement une ensemble de technique particulièrement adapté à la problématique de la durabilité de l'agriculture pluviale, les difficultés de sa mise en œuvre, non seulement dans les premières années de la phase d'apprentissage mais finalement sur le moyen terme (10 ans) pose la question de savoir si l'innovation proposée s'inscrit effectivement dans les conditions socio-économiques locales de la production (dans une logique très « schumpéterienne »).

Le projet a eu des effets bénéfiques évidents mais il a aussi créé des relations fortes entre projets et paysans qui ont créé un vide à l'arrêt du projet. Si l'autonomisation des acteurs a été une priorité du projet à partir de 2006, les outils mis en œuvre pour y arriver ont aussi contribué à créer une dépendance avec la relation particulière avec le technicien que nous retrouvons dans notre typologie comportementale.

\footnotetext{
${ }^{5}$ Une enquête menée en 20120 par Julie Sorèze pour le projet BVPI-SE-HP a montré que suite aux actions typiquement diffusionnistes de cette ONG, il ne restait absolument rien en 2010 et aucun paysan en AC n'a pu être retrouvé.
} 


\section{Des comportements contrastés après l'arrêt du projet}

Pour mettre en évidence l'attitude plus ou moins active des paysans face à l'adoption des techniques de l'AC et en condition post-projet, une typologie de comportement est réalisée et est établie sur les critères d'autonomie vis-à-vis de la connaissance des techniques et du besoin ressenti du projet. La psychologie particulière des paysans et le contexte amènent à des comportements nettement différenciés chez les anciens encadrés : i) 40\% sont « autonomes » et ont clairement annoncé qu'ils ne perçoivent pas de changement avec l'arrêt du projet dans leur façon d'appliquer les techniques de l'AC. Ils sont capables de gérer seuls leurs choix techniques. Ils ont, en général, augmenté leurs surfaces en SCV depuis 2009, ii) 30\% sont des « demandeurs » : ils se sentent autonomes mais ont exprimé le besoin de recevoir une aide technique pour les aider à gérer leur culture. Cette catégorie privilégie toujours le «contact» avec les projets et les techniciens dans une optique de valoriser au mieux l'information technique à laquelle ils peuvent avoir accès et iii) 30\% sont des « assistés » car ils éprouvent le besoin permanent d'une aide technique. Pour ces producteurs, il semble impossible de concevoir une adoption permanente d'innovation comme l'AC sans une aide technique, morale, matérielle ou financière. Ces sont ces paysans qui ont diminué leurs surfaces en $\mathrm{AC}$ et même abandonné pour la plupart (81\%).

Même si les paysans continuant effectivement les systèmes en AC en semblent satisfaits, la quasitotalité n'a adopté qu'un seul système de culture les rendant potentiellement plus fragiles techniquement que les paysans ayant diversifiés largement leurs systèmes. Ces résultats montrent que l'apparente cohésion sociale créée par le projet s'est effondrée plus rapidement que prévue et plus profondément que le besoin réellement ressenti en conseil technique : il reste $40 \%$ de paysans réellement autonomes. Le tableau 2 présente les points positifs et négatifs de l'adoption perçus par les producteurs enquêtés.

Le croisement de la typologie de situation avec la typologie de comportement a permis de comprendre en partie l'évolution (figure 6). Les paysans ayant un comportement «d'assisté » diminuent voire abandonnent ( $81 \%$ abandonnent). Ces paysans sont des paysans ayant impérativement besoin d'un appui technique pour continuer la pratique en AC. Ils ne se sont pas appropriés les techniques soit parce qu'ils suivaient à la lettre les consignes des techniciens soit parce qu'ils ne pratiquaient l'AC que pour bénéficier des avantages permis par le projet (produits phytosanitaires à moindre coût, kits de semences, établissement du Plan de Travail Annuel, sécurisation des parcelles...). Ces paysans ont tous arrêté les techniques AC lors du changement d'axe du projet (passage approche exploitation, conseil de gestion en 2010) ou à l'arrêt du projet (2013).

Un autre groupe est constitué de paysans ayant annoncé clairement leur autonomie. La majorité d'entre eux (20/29) ont augmenté leurs surfaces, ou ont des surfaces en AC qui représentent la quasitotalité de leur surface potentielle. Ces paysans « autonomes» ont su s'approprier les techniques. Ils ont donc pu, même en l'absence du technicien, garder une part importante de cultures en $\mathrm{AC}$ au sein de l'exploitation.

Enfin, le groupe des « demandeurs » est constitué par tous les types de situation d'exploitation. En effet, quel que soit leur type de situation, ces paysans peuvent vouloir un conseil technique même s'il n'est pas en lien avec les techniques en AC car les techniciens du projet intervenaient parfois aussi dans d'autres domaines (traitements contre les maladies du riz, techniques sur rizières, etc.). 


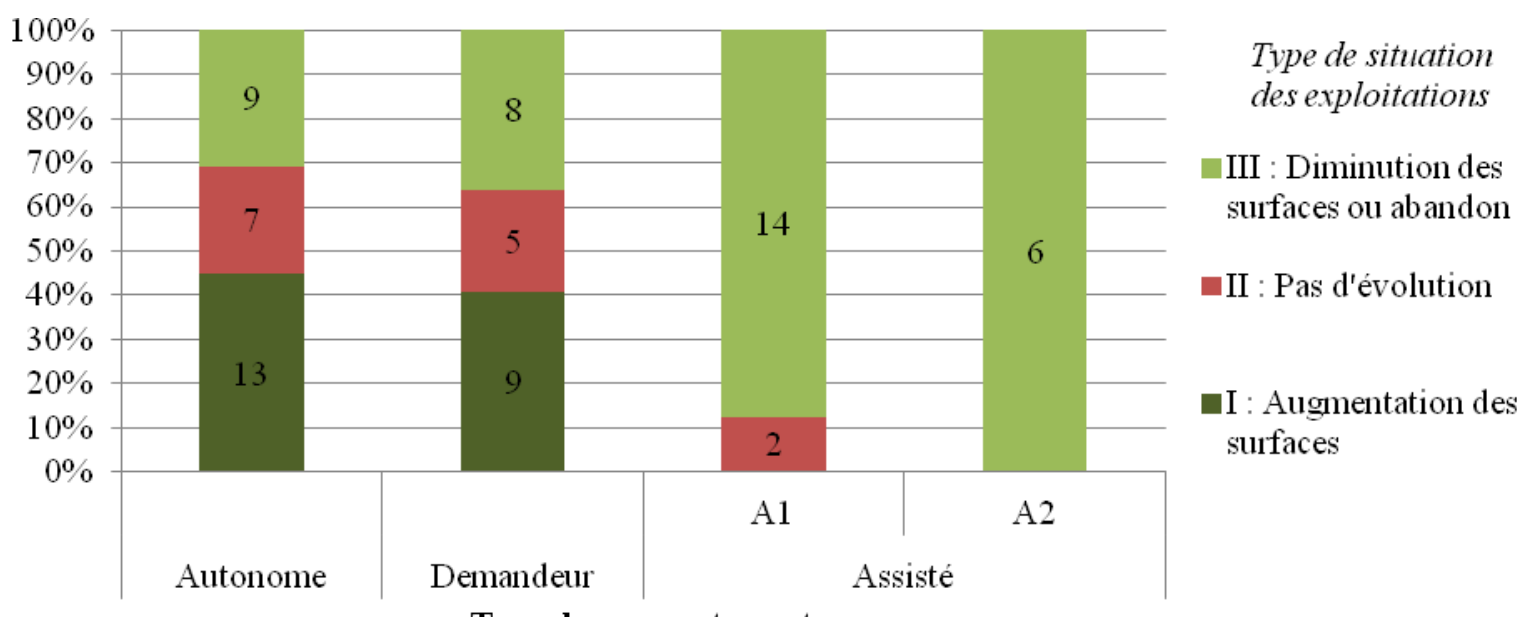

Typ e de comp ortement

Figure 6. Croisement entre la typologie de comportement et la typologie de situation 


\begin{tabular}{|c|c|c|}
\hline & $\begin{array}{l}\text { Points positifs permis par la } \\
\text { diffusion des techniques SCV }\end{array}$ & $\begin{array}{c}\text { Difficultés/obstacles pour l'adoption } \\
\text { durable des techniques SCV }\end{array}$ \\
\hline $\begin{array}{l}\text { Au niveau des } \\
\text { systèmes de } \\
\text { culture }\end{array}$ & $\begin{array}{l}\text { - Forte diminution des labours } \\
\text { - Rotation de cultures } \\
\text { - Large diffusion des SCI } \\
\text { - Intensification en nombre de culture } \\
\text { par cycle cultural (associations de } \\
\text { plantes productives, etc) } \\
\text { - Plantation en ligne }\end{array}$ & $\begin{array}{l}\text { - Les } 3 \text { principes de l'AC sont } \\
\text { rarement tous respectés } \\
\text { - Gestion de la couverture } \\
\text { - Gestion des semences de plantes de } \\
\text { service } \\
\text { - Compréhension du rôle de } \\
\text { l'association de plantes }\end{array}$ \\
\hline $\begin{array}{l}\text { Au niveau de } \\
\text { l'exploitation }\end{array}$ & $\begin{array}{l}\text { - Vision stratégique au sein de } \\
\text { l'exploitation par le raisonnement } \\
\text { des rotations } \\
\text { - Suppression des jachères } \\
\text { - Moins de fumier sur parcelles SCV } \\
\Rightarrow \text { économie pour parcelles non } \\
\text { SCV } \\
\text { - Meilleure gestion de l'exploitation } \\
\text { grâce à la tenue d'un cahier } \\
\text { d'exploitation }\end{array}$ & $\begin{array}{l}\text { - Pas de réelle intégration } \\
\text { agriculture/élevage avec les SCV à } \\
1^{\text {ere }} \text { vue } \\
\text { - Diffusion rare des SCV sur la totalité } \\
\text { des surfaces potentielle de } \\
\text { l'exploitation } \\
\text { - Stratégie de minimisation des risques } \\
\text { entrainant des difficultés pour la } \\
\text { diffusion des SCV sur tanety }\end{array}$ \\
\hline $\begin{array}{l}\text { Au niveau } \\
\text { territorial et } \\
\text { social }\end{array}$ & $\begin{array}{l}\text { - Entraide entre producteurs SCV } \\
\text { (VSE) } \\
\text { - Diffusion d'une partie des techniques } \\
\text { SCV des paysans encadrés vers les } \\
\text { non-encadrés } \\
\text { - 40\% de paysans réellement } \\
\text { autonomes }\end{array}$ & $\begin{array}{l}\text { - Respect des règles sociales } \\
\text { (problème de divagation des animaux } \\
\text { et accès au crédit) } \\
\text { - Dissolution des associations de } \\
\text { producteurs avec l'arrêt du projet } \\
\text { - Manque d'organisation paysanne } \\
\text { pour la disponibilité en semences } \\
\text { - 30\% de paysans non autonomes : } \\
\text { l'approche techniciste a renforcé le } \\
\text { caractère assisté }\end{array}$ \\
\hline
\end{tabular}

Tableau 2. Bilan des points positifs et négatifs sur l'adoption des techniques en AC

\section{Conclusion}

Un «coeur d'adoptant des techniques en $\mathrm{AC}$ » existe dans la région du lac Alaotra mais est apparemment beaucoup plus faible que celui attendu : ce sont des paysans réellement autonomes qui ont adopté durablement les techniques, convaincus de leurs efficacités pour leur exploitation et 
l'environnement, notamment pour la lutte contre l'érosion. Ils ont compris et apprécié le changement d'approche avec le passage au conseil de gestion et il semble que, pour eux, le changement de " paradigme » ait réellement eu lieu. Au vu du temps et du capital investis dans ce projet, il semble que le résultat soit peu significatif en termes d'adoption définitive de l'AC stricto sensu (du moins en 2014 date de cette étude) et il est encore bien trop tôt pour juger de la durabilité de l'adoption de l'AC. Un tel changement de paradigme montre bien l'importance du type de diffusion, du temps d'apprentissage nécessaire (au moins 7 ans) et de la nécessité de construire des formes de dissémination en partenariat générant une meilleure résilience en fin de projet (partenariat divers, plate formes d'innovations, etc ..) et qui reposent sur des bases sociales localement reconnues et non sur des OP crées ex nihilo par des structures de projet. L'utilisation « d'universités paysannes » comme cela a été fait dans le Nordeste du Brésil aurait permis par exemple l'émergence de groupements locaux socialement plus solides et probablement plus résilients dans le temps ${ }^{6}$.

Cependant, l'impact du projet ne se mesure pas qu'aux surfaces appliquant les trois principes de l'AC. En effet, les SCI semblent beaucoup plus développées à l'échelle du lac. Ce sont ces techniques qui paraissent finalement se diffuser aussi chez des producteurs qui n'étaient pas encadrés par le projet. Ces techniques répondent à de nombreuses contraintes des exploitants. En effet, tout en étant moins contraignantes que les systèmes en $\mathrm{AC}$, elles permettent de labourer moins souvent qu'en conventionnel, de maintenir une certaine humidité du sol avec la couverture, d'augmenter le revenu par l'augmentation du nombre de cultures rémunératrices dans la rotation, et d'utiliser moins d'engrais grâce à la couverture qui joue alors plus le rôle d'engrais vert que de plante de service. Cependant, ces systèmes SCI n'ont pas forcément les bénéfices environnementaux attendus de l'AC sur l'environnement (limitation de l'érosion, augmentation de la fertilité des sols, puits de carbone) par l'insuffisance, voire l'absence récurrente du mulch impliquant souvent un labour et sont donc probablement moins durables que les systèmes en AC. Ils sont néanmoins plus facilement adoptables et apportent une stabilisation, si ce n'est une augmentation des résultats économiques, ce qui est primordiale pour les paysans et constituent alors une forme de durabilité.

Ainsi, il est probable qu'à moyen terme les systèmes en AC stricto sensu ne perdurent pas tels qu'ils ont été initialement promus. Bien que le projet BV-Lac ait créé des conditions favorables à la dissémination des techniques en AC, l'approche techniciste initiale, les faibles résultats en termes de structuration effective des producteurs (montrant par la même la faible cohésion sociale des sociétés rurales locales) (PENOT et al., 2015) montre la nécessité de rechercher d'autres formes de diffusion avec une démarche participative, ou partenariale, et active des paysans ce qui n'était pas le cas avec l'approche top-down initiale.

L'approche techniciste, en lien avec la personnalité et le comportement social de certains paysans, a créé un comportement «d'assisté » pour 30\% des anciens encadrés, alors que les « autonomes » sont nettement plus nombreux chez les paysans n'ayant connu que l'approche conseil de gestion entre 2010 et 2012 comme l'a montré une autre étude réalisée à la même période (FEVRE et FLODROPS 2013). Ainsi, un développement basé sur l'utilisation de « courtiers de développement 》 (paysans alphabétisés ayant suivi de nombreuses formations et comprenant le discours des agents de développement ; BLANC-PAMARD et FOUROUX 2004) et sur une approche partenariale pourrait permettre un taux d'adoption à long terme des techniques de l'agriculture de conservation plus important, en tenant

\footnotetext{
${ }^{6}$ Cette option a été proposée en 2010 mais le bailleur de fonds n'a hélas pas donné suite.
} 
compte clairement de toutes les contraintes économiques et surtout sociologiques qu'amène un changement de paradigme aussi important que l'AC.

Il reste à mesurer l'impact réel du projet en termes de diffusion des techniques agro-écologiques au sens large et leur contribution réelle à la durabilité de l'agriculture pluviale locale. Nul doute que la plupart des techniques de l'AC seront plus ou moins intégrées aux systèmes de culture de culture locaux comme on a pu le mesurer en 2010 sur les techniques améliorantes introduites dans les années 1980 par le projet R-D au lac Alaotra (FOFIFA/CIRAD). Cette recombinaison des savoirs montre une véritable économie de la connaissance (LAPERCHE, 2008) qui est au moins aussi importante que l'économie du développement. L'analyse des processus d'apprentissage apporte autant pour la compréhension de la durabilité des processus que l'information classique sur l'économie de $1^{\prime}$ 'exploitation agricole censée susciter des comportements économiques. L'exemple de notre typologie comportementale montre que les facteurs décisionnels ne sont pas seulement économiques ou techniques mais sociaux ou les formes d'apprentissage relèvent de stratégies certes individuelle (du moins dans le cadre de notre exemple au lac Alaotra) mais aussi inscrite dans un collectif particulièrement difficile à cerner et à comprendre, pourtant essentiel à intégrer dans la compréhension des processus d'innovation. Si l'évolution des systèmes techniques est relativement facile à identifier, les raisons profondes de cette évolution, même avec une bonne périodisation historique et l'identification des contextes qui ont permis l'émergence de telle innovation, restent souvent difficiles à cerner dans le moyen et long terme (entre 10 et 30 ans).

La mise en place d'un fonds régional local de développement est prévue en 2016/2017 au lac Alaotra qui permettrait de financer des demandes locales ce qui implique que les acteurs de la production (et de la commercialisation) puissent avoir la capacité d'identifier puis de problématiser leurs demandes avec cette fois non plus la position de « client » d'un projet mais de véritable partenaire du développement local. La co-construction de modèles techniques adaptés est cruciale pour le développement futur de cette zone ou la population double tous les 18 ans. La co-évolution des systèmes techniques (TOUZARD et al., 2015), initiée par le projet BV6lac, en devenir, et la connaissance fine des mécanismes d'innovation sur le moyen terme comme celle proposée dans cet article deviennent alors des notions clés pour assurer un développement durable. Ainsi, la création d'un système d'innovation sectoriel futur centré sur le développement de pratiques agro-écologiques dépendra de la réalité effective du réseau socio-technique (TOUZARD, 2014) précédemment créé par la rencontre entre le projet BV-lac et des acteurs de la production en demande de solutions techniques pour une agriculture durable qui soient socialement intégrables dans leurs systèmes d'activités. 


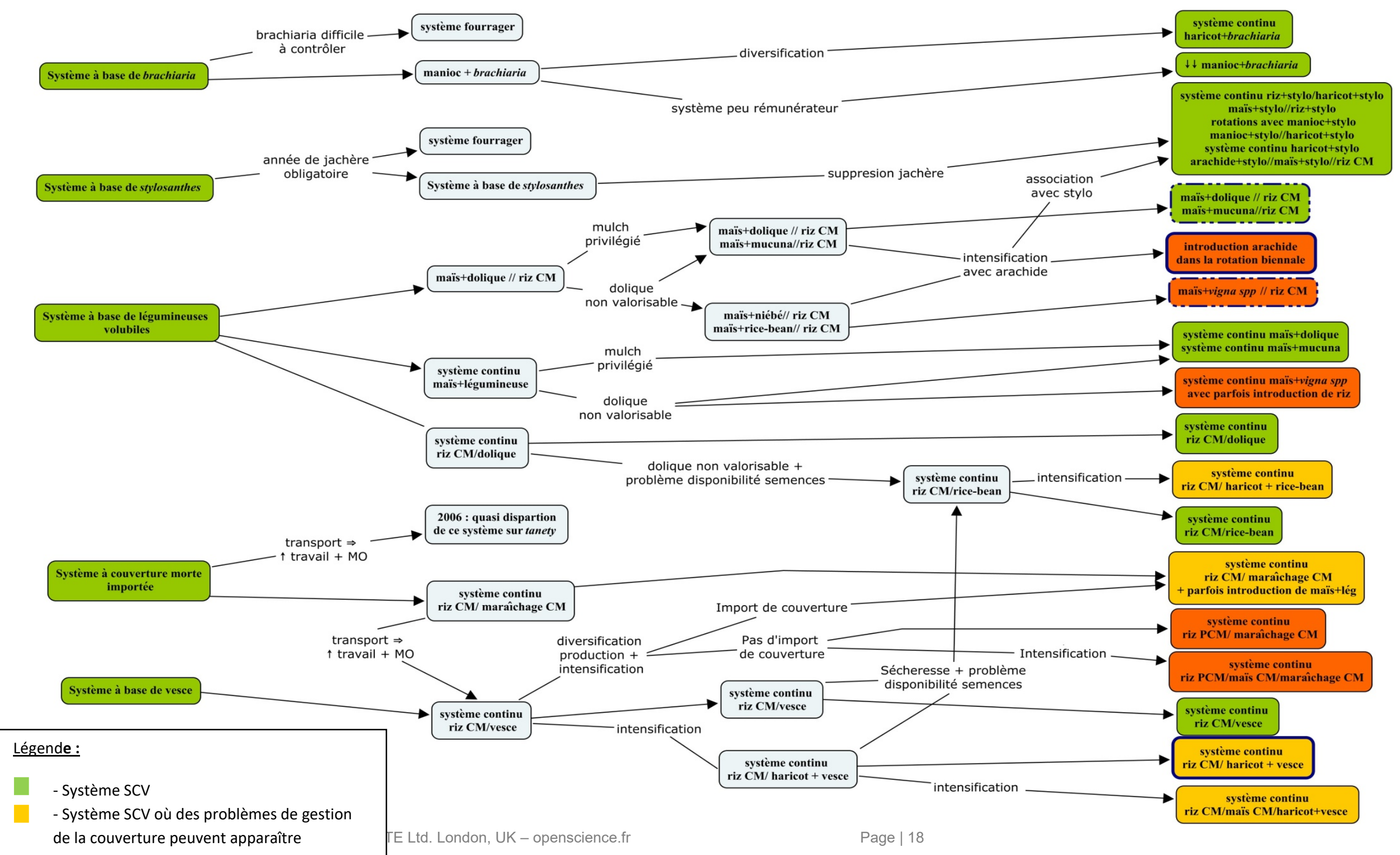

- Système $\mathrm{SCl}$ ou proches $\mathrm{SCl}$

$\square$ - Systèmes majoritaires

!- ! - Deuxièmes systèmes majoritaires

Figure 3. Evolution des systèmes diffusés depuis 2003 jusqu'à 2013 à partir des grandes familles de systèmes 


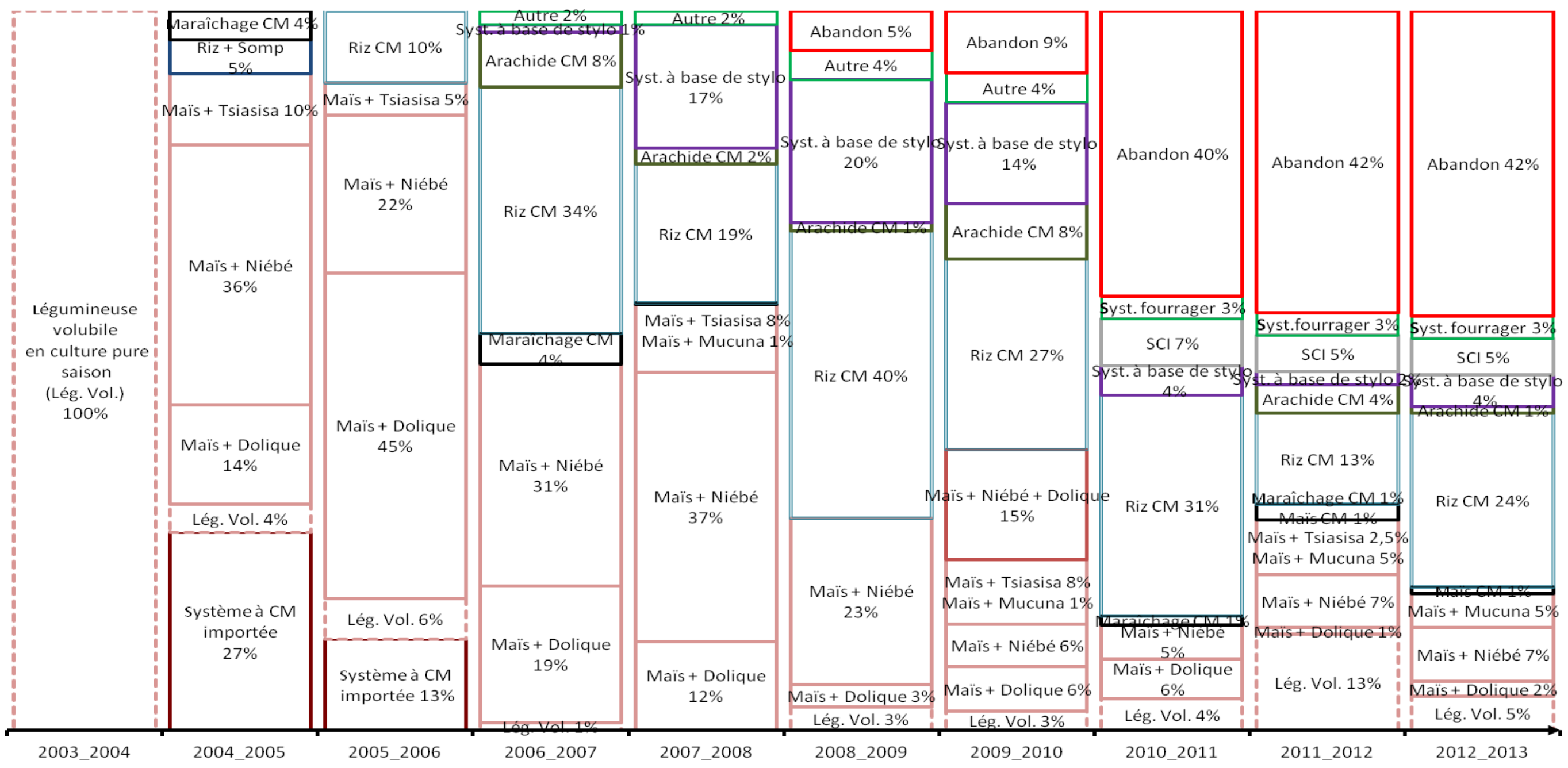

Figure 4. Evolution des surfaces ayant débutées par une légumineuse volubile en culture pure ou associée au maïs sur tanety 
BLANC-PAMARD MC \& FOUROUX E., L'illusion participative. Autrepart, (3), p. 3-19, 2004.

COTTET, L. \& PENOT, E. (2010). Mise au point des scénarios d'analyse prospective développés sur les réseaux de fermes de référence (RFR) au sein du Projet BV Lac Alaotra: modélisation et préparation des recommandations techniques et plans de campagne PTA pour 2010. Rapport BV-lac. 2010. $101 \mathrm{p}$.

CORBEELS M, DE GRAAFF J, HYCENTH NDAH T, PENOT E, BAUDROND F, NAUDIN K, ANDRIEU N, CHIRAT G, SCHULER J, NYAGUMBO I, RUSINAMHODZIB L, TRAORE K, DULLA MZOBAG H, SOLOMON ADOLWAH I., Understanding the impact and adoption of conservation agriculture in Africa: A multi-scale analysis. Agriculture, Ecosystems and Environment 187, p 155-170, 2014.

COUDEl E., DEVAuTOUR H., SOUlARD T., FAURE G., HUBERT B., Apprendre à innover dans un monde incertain, Versailles, QUAE, 2012.

DOMAS R., PENOT E.,ANDRIAMALALA H., CHABIERSKI S. Quand les tanety rejoignent les rizières au lac Alaotra : diversification et innovation sur les zones exondées dans un contexte foncier de plus en plus saturé. Regional workshop on conservation agriculture, CIRAD/AFD, Phonsavan Xieng Khouang Laos PDR, 31p, 2009.

DURAND C., NAVE S., PENOT E., Les paysans de l'Alaotra, entre rizières et tanety - Étude des dynamiques agraires et des stratégies paysannes dans un contexte de pression foncière - Lac Alaotra, Madagascar. Document de travail BV lac/AFD., $38 \mathrm{pp}$.

DULCIRE M., Le jeu de l'implication et le feu de l'engagement: chroniques nicaraguayennes. Économie rurale, 236, p. 62-68, 1996.

DULCIRE M, CHIA E, VALL E, Conception des Innovations et rôle du partenariat, CIROP. Bilan et perspectives des activités et résultats. Montpellier: CIRAD, 2008.

FABRE J. Evaluation technico-économique des effets des systèmes de culture sous couverture végétale dans les exploitations agricoles du lac Alaotra, Madagascar. Mémoire master, Montpellier, SupAgro, 162p , 2011.

FAURE G, PENOT E, RAKOTONDRAVELO JC, ANDRISOA RAMAHATORAKA H, TOILLIER A., Which Advisory System to Support Innovation in Conservation Agriculture? The Case of Madagascar's Lake Alaotra. The Journal of Agricultural Education and Extension. International Farming Systems Association Symposium. Vol.19, $\mathrm{n}^{\circ}$ 2013, p. 257-270. DOI:10.1080/1389224X.2013.782169

FAO. Investing in Sustainable Agricultural Intensification. The Role of Conservation Agriculture. A Framework for Action. Food and Agriculture Organization of the United Nations, Rome, 2008.

GARIN P, PENOT E. Charrues et variétés de riz. Maîtrise sociale des savoir-faire techniques au Lac Alaotra, Madagascar. Revue d'anthropologie des connaissances, 5 (3), p. 573-598. http://www.cairn.info/revue-anthropologiedes-connaissances, 2011

GILLER K, WITTER E, CORBEELS M AND TITTONELL P., Conservation agriculture and smallholder farming in Africa: The heretics' view. Field Crops Research 114, p. 23-34, 2009.

LAPERCHE B., L'innovation pour le dévelopement. Enejux globaux et opportunités locales. Karthala. Paris. 255 p, 2008.

MAC DOWALL, C.,Socio-economic assessment of the impact of adoption of conservation agriculture on farming systems in the region of lake Alaotra, Madagascar. Mémoire de master en agroéconomie, ISARA-Lyon, CIRAD, $127 \mathrm{p}, 2011$.

PENOT E, DABAT MH, RAKOTOARIMANANA A, GRANDJEAN PH., L'évolution des pratiques agricoles au lac Alaotra à Madagascar. Une approche par les temporalités Biotechnol. Agron. Soc. Environ 18(3), pp. 329-338, 2014

PENOT E, BENZ H, BAR M., Utilisation d'indicateurs économiques pertinents pour l'évaluation des systèmes de production agricoles en termes de résilience, vulnérabilité et durabilité : le cas de la région du lac Alaotra à Madagascar Éthique et économique, 11 (1), 2014. http://ethique-economique.net/.

PENOT E, DOMAS R., Rôle et place du riz pluvial dans les exploitations agricoles à Madagascar. Le cas du Lac Alaotra. Académie d'Agriculture, , pp.1-30, 2011

PENOT E., FABRE J AND DOMAS R., The real adoption of conservation agriculture (CA) in the lake Alaotra area after 10 years of diffusion. [Poster]. Projet ANR pépites In: 5th World Congress of Conservation Agriculture (WCCA), Brisbane, Australia, 2011. DOI 2011-09-26/2011-09-29. www.wcca2011.org 
PENOT E, DOMAS R, FABRE J, POLETTI S, MACDOWALL C, DUGUE P, LE GAL PY., Le technicien propose, le paysan dispose. Le cas de l'adoption des systèmes de culture sous couverture végétale au lac Alaotra, Madagascar. Cah. Agric. 24, p 84-92, 2015.

ROGERS EM., 1962. Diffusion of Innovation. 4rth edition. Free Press NYC, USA. 1995.

SCOPEL E., TRIOMPHE B., DE FATIMA M., DOS SANTOS R., SEGUY L., DENARDIN EJ., KOCHHANN RA., Direct seeding mulch-based cropping systems (DMC) in Latin America. New directions for a diverse planet, p.1-16, 2004.

SCOPEL E., TRIOMPHE B., AFFHOLDER F., DA SILVA F.A., CORBEELS M., VALADARES XAVIER J.H., Conservation agriculture cropping systems in temperate and tropical conditions, performances and impacts. Agronomy for Sustainable Developemnt 33, p.113-30, 2012.

SERPANTIE G., L'agriculture de conservation à la croisée des chemins. Vertigo, $9: 21$ p, 2009.

TEISSONNIER, A. Analyse des modes d'appropriation et d'adoption des techniques de l'agriculture de conservation hors projet dans la zone du lac Alaotra, Madagascar. Rapport de stage de césure, Cleront-Ferrand, VetAgroSup, 71p, 2013.

TOUZARD JM., Les approches sectorielles de l'innovation. Chapitre 16. In "Les principes d'économie de l'innovation". 2014. PIE Peter Lang. Collection Business \& information $n^{\circ}$ 8. Pp. 235-246, 2014.

TOUZARD JM, TEMPLE L., FAURE G., TRIOMPHE B..,«Innovation systems and knowledge communities in the agriculture and agrifood sector: a literature review», Journal of Innovation Economics \& Management. Vol 2, $\mathrm{n}^{\circ} 17, \mathrm{p}$. 117-142. 2015. DOI 10.3917/jie.017.0117

URFER S., Ou va Madagascar? Les incertitudes d'une société en mutation. Etudes. Ed Cairn. 10 (octobre). p 19-27, 2014.

VALENTIN FEVRE, FLODROPS P., Comparaison sur l'adoption des techniques de l'Agriculture de Conservation pour différents groupes de paysans encadrés à différentes époques dans la région du Lac Alaotra. Mémoire de stage de césure Agroparistech. 30 p, 2013. 